\title{
Stellar evolution, GRB and their hosts
}

\author{
I.E. Panchenko ${ }^{1}$, V.M. Lipunov ${ }^{1,2}$, K.A. Postnov $^{1}$, and M.E. Prokhorov ${ }^{2}$ \\ 1 Faculty of Physics, Moscow State University, 119899 Moscow, Russia \\ 2 Sternberg Astronomical Institute, Moscow State University, 119899 Moscow, Russia
}

Received January 21; accepted June 1, 1999

\begin{abstract}
We compute the GRB rates in different types of galaxies by performing a population synthesis using the "Scenario Machine" code in the framework of merging binary relativistic stars model. Our model predicts that $\sim 1 / 3$ of GRB should occur in elliptical galaxies, and this fraction should increase with the redshift. This fraction is comparable with the observed fraction of GRB without (detectable) X-ray afterglows.
\end{abstract}

Key words: gamma-rays: bursts

\section{Motivation}

The binary neutron star mergers ( Blinnikov et al. 1994; Paczyński 1991) are one of the most probable GRB progenitors. Several works were devoted to fitting the observed parameters of GRB (mainly, $\log N-\log P$ distributions) by evolutionary models (e.g. Fenimore et al. 1993; Jørgensen et al. 1995; Totani 1997; Bagot et al. 1998). It is often supposed in such calculations that the merger rate is proportional to the star formation rate.

However, the coalescence time of the $\mathrm{NS}+\mathrm{NS}$ and $\mathrm{NS}+\mathrm{BH}$ binaries can be very long, and some of the mergers should take place in rather old stellar population, even in elliptical galaxies where there should be no noticeable star formation. This property can help to distingwish the binary merger GRB from the GRB from massive star collapses.

\section{The model}

In our previous paper (Jørgensen et al. 1995) we had computed by population synthesis the NS+NS coalescence time distribution. This allowed us to obtained a good fit to the observed $\log N-\log P$ distribution of the BATSE GRB. Transition from the coalescence time distribution to the $\log N-\log P$ requires knowledge of the star formation rate (SFR) history, which was approximated by a simple two-parametric model including an intensive burst (in which $\epsilon$ of all the stars were born, at a redshift $z_{\text {star }}$ ), and a subsequent constant SFR.

Since that time our knowledge of cosmic SFR history increased by the works of Madau et al. (1996) and others. Now it has become evident that the star formation rate is far from being constant and strongly rises with redshift up to $z \simeq 1-1.5$. To account for the realistic SFR, we used the cosmic SFR history taken from Madau et al. (1996). However, the SFR at higher redshifts $z>1.5$ is still poorly known, so we introduce an additional SFR burst at $z_{\star}=5$ to account for the early formation of the elliptical galaxies and spheroidal components of the spiral galaxies. The fraction of the stellar matter formed in this burst we denote by $\epsilon$. We attribute the star formation burst to the galactic spheroidals and the subsequent star formation - to the galactic disks. The ratio of the amount of the visible matter in galactic disks and spheroidals is $\sim 1 / 2$ (Fukugita et al. 1998), which corresponds to our $\epsilon=2 / 3$.

The coalescence time distributions were computed by the "Scenario Machine" population synthesis engine (Lipunov et al. 1996a, 1996b) with an ordinary set of model parameters. The stellar evolution model was based on the results of Vanbeveren et al. (1998), which reproduce most accurately the observed galactic WR star distributions and stellar wind mass loss in massive stars.

As the main parameter influencing the result was the kick velocity, we display the result for several possible values of the mean kick velocity.

Figure 1 shows the coalescence time distributions for different values of mean kick. Note that the distributions have a power-law behaiviour, which implies a significant part of the coalescences time to be long. If there is no kick, the neutron star coalescence times are preferably long (there should be no coalescences in young stellar population), and the $\mathrm{NS}+\mathrm{BH}$ binaries coalescence times are even longer than the Hubble time, so the observable event rate should be negligible.

The coalescence time distributions can be used for calculating the GRB rates. As relative rates are easier 

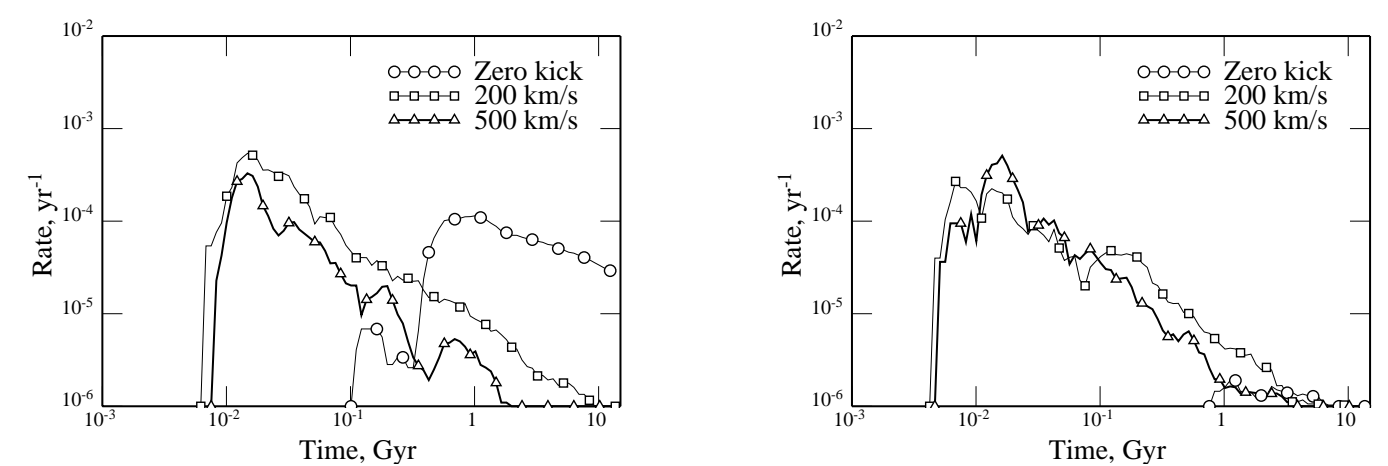

Fig. 1. Coalescence time distributions for different kick velocities normalized for a sample galaxy with instantaneous star formation of $10^{11} M_{\odot}$. Left: NS+NS. Right: NS+BH
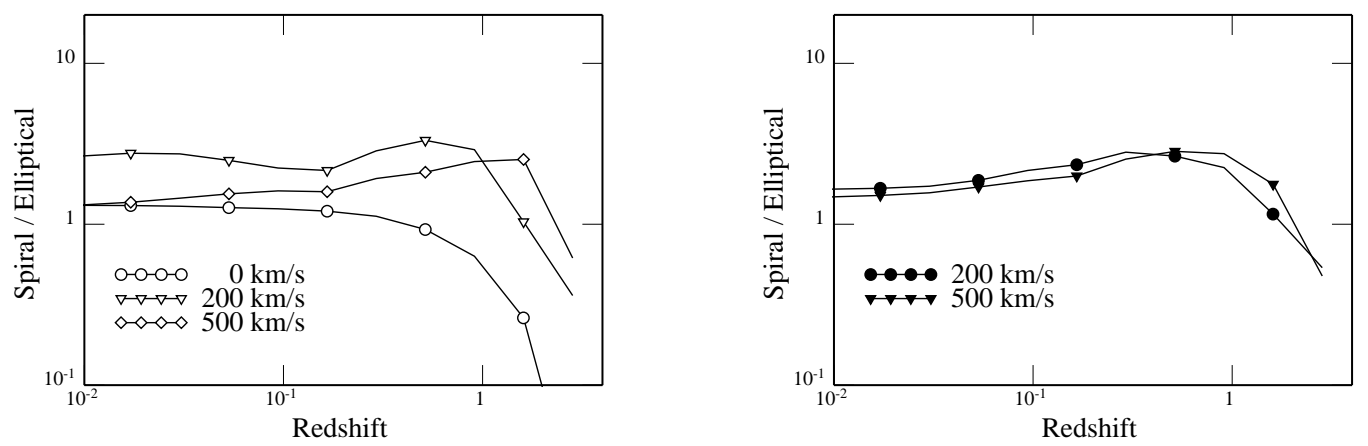

Fig. 2. The ratio of the coalescence rates in spiral and elliptical galaxies at different redshift for different kick velocities. a) Left panel: NS+NS. b) Right panel: NS+BH

to observe than the absolute ones, here we show (see Fig. 2) the relative rate of coalescences in spiral and elliptical galaxies, obtained in a standard cosmological model $\left(H_{0}=75 \mathrm{~km} \mathrm{~s}^{-1} / \mathrm{Mpc}, \Omega=1\right.$ and $\left.\Omega_{\Lambda}=0\right)$.

\section{Discussion}

The performed computations show that the moderate redshifts about $0.1-1$ where the most GRB are observed now, the rate of GRB in spiral galaxies should be $2-3$ times higher than in ellipticals (Fig. 2).

If GRB afterglow is produced by interaction of a relativistic shock wave with the interstellar medium (Mészáros \& Rees 1992), the afterglows in elliptical galaxies should be dimmer than in the spirals where the interstellar medium is more dense. Present observations of GRB afterglows show that approximately $1 / 4$ of the bursts with good localization do not have detectable X-ray afterglows, which is consistent with the expected fraction of GRB in elliptical galaxies.

Our model predicts an increase of relative number of the GRB in elliptical galaxies at high redshifts. A direct observational detection of an elliptical host galaxy of a GRB is desirable to support the binary model of GRB. ipation in the conference possible. This research was carried out under the support of INTAS project No. 96-0315 and the RFBR grant No. 98-02-16801.

\section{References}

Bagot P., Portegies Zwart S., Yungelson L.R., 1998, A\&A 332, L57

Blinnikov S.I., Novikov I.D., Perevodchikova T.V., Polnarev A.G., 1984, PAZh 10, 422; Sov. Astron. Lett. 10, 177

Fenimore E.E., et al., 1993, Nat 366, 40

Fukugita M., Hogan C.J., Peebles P.J.E., 1998, ApJ 501, 518 Jørgensen H.E., Lipunov V.M., Postnov K.A., Prokhorov M.E., Panchenko I.E., 1995, ApJ 454, 593

Lipunov V.M., Postnov K.A., Prokhorov M.E., 1996, A\&A 310, 489

Lipunov V.M., Postnov K.A., Prokhorov M.E., 1996, The scenario machine: Binary star population synthesis. Amsterdam: Harwood Academic Publishers

Madau P., et al., 1986, MNRAS 283, 1388

Mészáros P., Rees M.J., 1992, MNRAS 258, 41

Paczyński B., 1991, Acta Astr. 41, 257

Totani T., 1997, ApJ 486, L71

Vanbeveren D., et al., 1998, New Astron. 3, 443 